\title{
Digitization of Supply Chains as a Lever for Controlling Cash Flow Bullwhip: A Systematic Literature Review
}

\author{
Hicham Lamzaouek ${ }^{1}$, Hicham Drissi ${ }^{2}$, Naima El Haoud ${ }^{3}$ \\ Laboratory of Financial Engineering, Development and Governance (LIFGOD) \\ National School of Business and Management of Casablanca, Hassan II University, Casablanca 20250, Morocco ${ }^{1,2}$ \\ ENCG Casablanca/ Hassan 2 University, Casablanca, Morocco ${ }^{3}$
}

\begin{abstract}
Due to the new possibilities offered by digital technologies, more and more companies are embarking on a process of digitizing their supply chains. This dynamic seems to be the opportunity to analyse the impact that digital technologies may have on one of the phenomena that disrupt financial flows within supply chains, and that can alter the companies' treasury, namely that of cash flow bullwhip (CFB). The results of the systematic literature review that was carried out allow to affirm that several technologies can contribute positively to limiting this phenomenon and this by acting on these operational causes, which are the reliability of forecasts, batch orders, the fluctuation in sales prices, rationing games, and lead times.
\end{abstract}

Keywords-Cash flow bullwhip; digital technologies; digitization; supply chain; cash flow; bullwhip effect

\section{INTRODUCTION}

Without controlling its financial flows, the company cannot continue to operate even if it proposes an attractive offer to the market [1]. In this regard, it is obvious that any company must pay particular attention to this part of the flows which are in reality only the consequences of the physical and information flows of a supply chain. In fact the overall performance of companies is linked with their financial performance [2], [3], which make the control of financial flows very important. That said, research has shown that these financial flows are usually undergoing a disruption, referred to as cash flow bullwhip (CFB), and which is observed in almost all supply chains [4]. In this regard, it has been shown that CFB originates from the phenomenon of the bullwhip effect [4]. Indeed, the amplification of stocks caused by the BWE, slows down the time necessary to convert them in sales, and even makes this time instable. This leads to a delay in the cash flow generation [4].

The diagram below illustrates the dynamics of the CFB at the level of the supply chain (Fig. 1).

Mathematical modeling of the CFB shows that this disturbance is caused by the bullwhip effect and supply chains' lead times [4]. This is to say that the causes of CFB are lead times, reliability of sales forecasts (quality of demand signals processing), batch orders, sales price fluctuations, and rationing games between supply chain members [5].

While several studies have tried to propose solutions for the causes listed above [6], the rise of digital technologies prompts to be interested in the possible contributions of these technologies in reducing the causes of CFB. In what follows, we propose a systematic review of the literature concerning the contribution of digital technologies to the control of CFB. We propose to structure the rest of the document in three main parts. The first part will be devoted to the research methodology. The second will present the descriptive analysis of the results. The third part will synthesize the results of the content analysis by identifying the concept of digitization and the main digital technologies, and by illustrating the impacts of these technologies on each of the operational causes of CFB. We will conclude with a summary and avenues for further research.

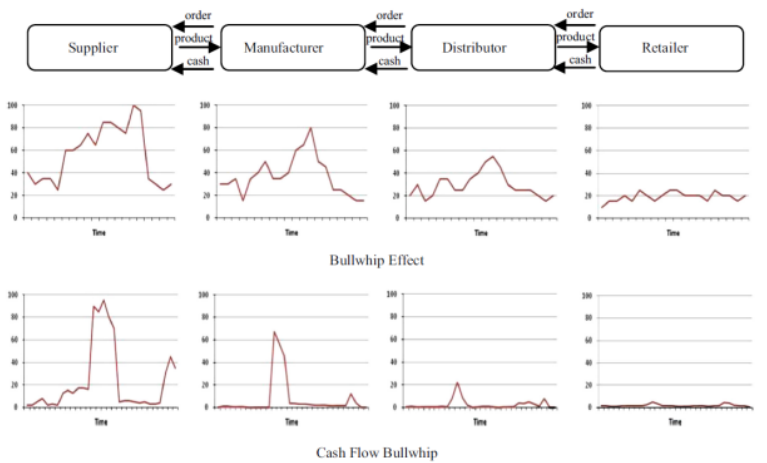

Fig. 1. BWE and CFB in Supply Chains (Tangsucheeva \& Prabhu, 2014).

\section{MethodOlOGY}

In order to fully understand the potential impacts of digitalization on the $\mathrm{CFB}$, we opt for a systematic literature review. This type of literature review is more methodical compared to narrative reviews, and establishes an in-depth description of the steps taken to select, examine and analyze relevant sources with the aim of minimizing bias and increasing transparency.

We choose the approach of Denyer and Tranfield which distinguishes the following four steps (Fig. 2):

The research question can be formulated as following:

- How the use of digital technologies by supply chain members, can lead to a control of the cash flow bullwhip? 


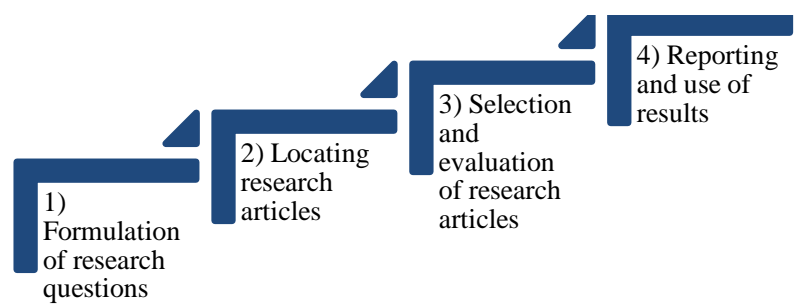

Fig. 2. Research Methodology.

To answer this question, we need to first address the following questions:

- What is the meaning of digitization?

- What are the main digital technologies related to supply chain management?

- In which manner can the digital technologies act on the operational causes of cash flow bullwhip?

\section{A. Definition of Keywords and Databases}

Articles potentially related to our topic were identified in the "Scopus" and "Taylor \& Francis" databases by using the combination of the following terms: "Digital technologies and cash flow bullwhip", "Digital technologies and bullwhip effect", "Digitization and cash flow bullwhip", "Digitization and bullwhip effect".

\section{B. Definition of Selection Criteria}

The identification of the most relevant articles was carried out in October 2020. The selection was made based on reading article summaries or book introductions. The diagram below shows the item selection process (Fig. 3):

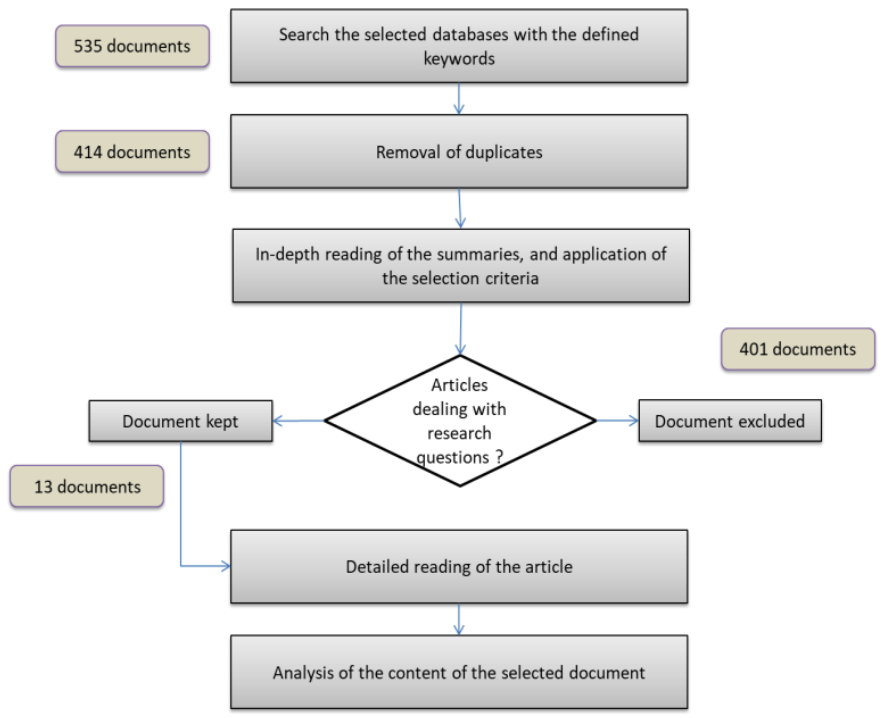

Fig. 3. Research Work Selection Process.

\section{DESCRIPTIVE ANALYSIS OF THE RESUltS}

By analyzing the nature of the research work selected, we find that most of them are journal articles, as the figure below shows (Fig. 4):

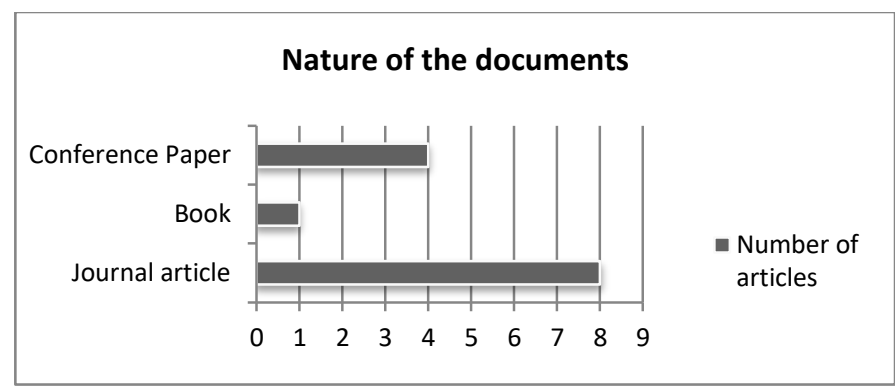

Fig. 4. Number of Documents per Nature.

On the other hand, the analysis of research work publication's year shows that this is a relatively new subject, since the first article identified was released in 2005. The figure below shows the distribution of the articles, by year of publication (Fig. 5):

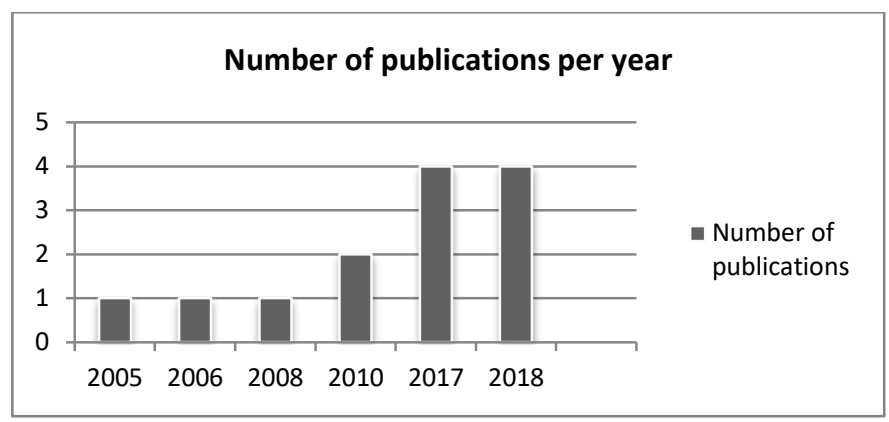

Fig. 5. Distribution of Publications per Year.

Finally, the analysis of the research work type reveals the following results (Fig. 6):

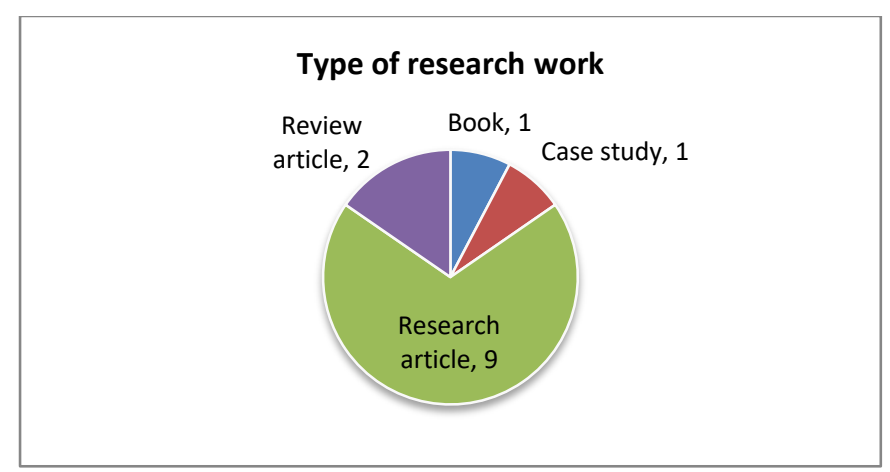

Fig. 6. Distribution of Research Work by Type.

\section{CONTENT Analysis Results}

\section{A. Digitizing}

Looking at the existing literature, several definitions can be associated with digitization. For some authors, digitalization is the integration of digital technologies into business processes [7][8]. For others, it is an organizational strategy deployed using digital resources to create value [9]. A third definition that seems adequate is that digitizing represents a fundamental change in traditional business practices by redefining new capabilities, processes, and relationships [10]. 
Despite the diversity of existing definitions, these converge towards the fact that digitization is not limited to the use of digital technologies, but also involves a break with traditional business processes and relationships based on capabilities digital, thus transforming the business model, operational processes and customer experience.

\section{B. Digital Technologies}

Several works have tried to list and identify the digital technologies that constitute the basic foundation of digitization. We propose to limit ourselves to the framework proposed by Schlüter and Hetterscheid in 2017, which is oriented towards the digital technologies used at the level of supply chains, and which are as follows [11] (Fig. 7):

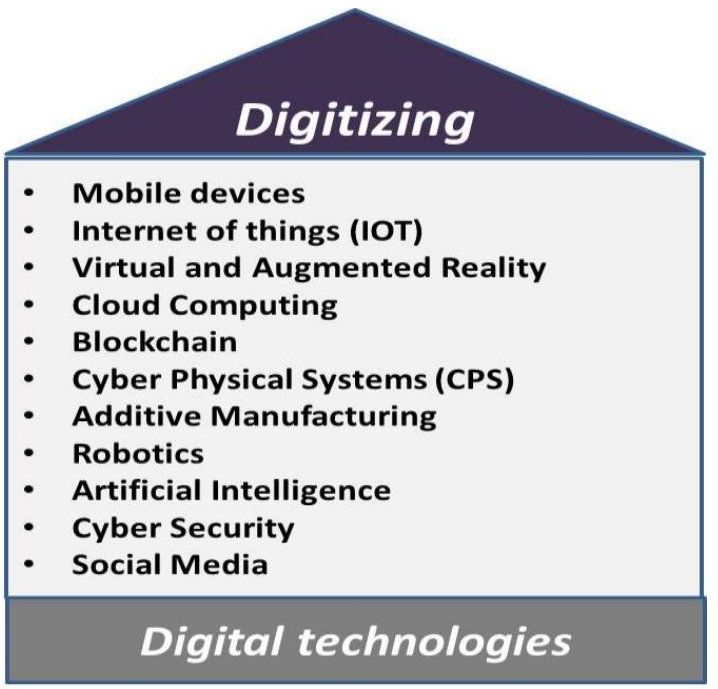

Fig. 7. Digital Technologies.

- Mobile devices: A mobile device is a portable tablet or other device designed for portability, and therefore is both compact and lightweight. It's powerful enough to do the same things as a computer.

- Internet of Things (IOT): It is a dynamic global network infrastructure with self-configuration capabilities based on standard and interoperable communication protocols where physical and virtual "objects" have identities, physical attributes and virtual personalities, use intelligent interfaces and are seamlessly integrated into the information network [12]. These infrastructures connect objects and make it possible to access, manage and exploit data. [13].

- Virtual reality is defined as the development of simulated expertise which is somewhat similar to the real-time situation. In addition, virtual reality is an imitation using the computer / communication devices across borders [14]. Augmented reality is a technology that allows you to mix between the virtual world and the real world. It allows digital information to be added to our actual visual field, by superimposition.

- Cloud Computing: A style of computing in which computing capabilities scale and are delivered as a service via internet technologies 'in the cloud'. It also refers to the use of software owned, delivered, and managed remotely by a third party on a paid or subscription basis (also referred to as "software as a service" or "SaaS") [15].

- Blockchain: This is a distributed ledger in which data is stored in a series of blocks. Each of the nodes in a blockchain network has a copy of the blockchain. Each time blockchain data is modified, it is distributed throughout the network [16]. Blockchain technology relies on distributed ledgers, encryption, Merkle tree hashing, and consensus protocols [17].

- Cyber-physical system (CPS): Systems, which directly connect real (physical) objects and processes to (virtual) information processing objects and processes via interconnected, open, and partially global information networks [18].

- Additive manufacturing: It is a process of creating physical objects by superimposing different layers of material on the basis of a digital model. [19].

- Robotics: A robot is a reprogrammable multifunctional manipulator designed to move equipment, parts, tools, or specialized devices by variable programmed movements to perform a variety of tasks. It is a machine capable of automatically performing a complex series of actions programmed by a computer.

- Artificial intelligence: is the means of exploiting, in real time, the mass of information collected, of sorting and analyzing it via algorithms making it possible to build predictive models. This can only be achieved with Big Data, which can be defined as a new generation of technologies designed to enable organizations to extract value from large volumes of data [20].

- Cyber Security: Cyber security corresponds to all the techniques used to preserve the integrity of networks, programs and data against unauthorized access. It refers to all technologies and processes and can also be referred to as information technology security [21].

- Social media: Corresponds to technologies that allow for social interactions, using communication capabilities, such as the internet or a mobile device [15].

\section{Impacts of Digital Technologies on the Operational Causes of $C F B$}

1) Demand forecast: One of the major causes behind the CFB is the reliability of demand forecasts and their updating. In fact, forecasts are often based on historical demand. However, distortions in demand can occur, for example, when a customer places an order, the supplier tends to treat this information as a signal of future demand for the product, but in reality the customer's demand often includes a part which is linked to a safety stock that the one always tries to keep in order to protect itself against the variation of demand and delivery times [5], [22]. In this regard, improved demand 
forecasting and processing of demand signals may be possible through the use of CPS which allows the sharing of operational production information in real time, improving visibility into physical flows [23].

Furthermore, by excluding human interpretation bias, artificial intelligence can improve the processing of demand signals. Indeed, through the volume of data offered by Big Data, it is easy to develop artificial intelligence to detect and interpret patterns, thus leading to better processing of demand signals [24].

On the other hand, improved processing of demand signals can be achieved using cloud computing. Indeed, by introducing the standards of communication, security and confidentiality, the cloud contributes to the reduction of data inconsistencies that serve as the basis for processing demand signals [25].

That said, another technology also improves the processing of demand signals. Thus, the demand forecasting, which is often carried out by each supply chain member without synchronization, can be significantly improved thanks to the speed of the transmission of information that RFID allows. Indeed, through the data stored at the tag level, RFID makes it possible to provide and process information more quickly and efficiently. This helps to give better visibility in the logistics chain in relation to the flow of materials. This makes integrated planning and therefore better decision making possible [26].

The impact of RFID on improving processing of demand signals has also been highlighted by other researchers. Indeed, the simulation work carried out on a logistics chain, shows that the elimination of the inaccuracy of stocks, which this technology allows, helps to avoid stock-outs [27].

In addition, blockchain is a digital technology that can help improve the processing of demand signals, thanks to the sharing of information it enables between actors in the supply chain. This is because as data sharing is distributed, members of a supply chain can have equal access to data from other members, even when they are further downstream [28].

2) Batch order: Grouping orders in batches can lead to information distortion. Indeed, since placing orders is generally expensive and time consuming, companies prefer to order in batches, instead of ordering their strict needs more frequently [5], [22]. On the other hand, at the production level, manufacturers set the sizes of production batches, to deal with the setup times required to adjust their production machines.

These optimization practices at the local level of each stakeholder in the chain, leads to inefficiency throughout the supply chain thus causing CFB. In this regard, several digital technologies offer solutions to the reduction of batch sizes by reducing the times of operations relating to setup and lunch times.

Thus, the deployment of RFID at the level of the production lines allows the automation of a certain number of operations which are part of the setup times, such as those dedicated to the establishment of tracking sheets, quality sheet, etc. RFID deployed in warehouses contributes to reducing the time and effort required to receive orders, as can be seen from the experience of the Metro distributor, which reduced the time it took to process pallets from 90 to 70 seconds through the implementation of RFID [26]. This encourages companies to reduce the size of replenishment order lots.

Another contribution of digital technologies in the reduction of batch sizes is the use of CPS systems for the automation of quality controls, which allows manufacturers to reduce the time dedicated to quality controls which are part of the setup time, and therefore reduce batch sizes [29].

3) Fluctuation in selling prices: Fluctuating prices have an impact on the purchasing decisions of customers. When the price of a product is reduced, customers can buy it in larger quantities than necessary [5], [22]. When the price of the product returns to normal, customers stop buying it until their stocks are depleted. Due to these price fluctuations, the customer's purchasing habits do not reflect an actual consumption pattern, and the variation in quantities purchased is much greater than that in the rate of consumption [5], [22].

This situation, which creates a desynchronization between the rhythm of the order and the rate of consumption of the products, thus gives the supplier an erroneous sign as to the real need of the market. This often causes the supplier to restock (produce) more to keep up with the momentary rise in demand. At the end of the promotions, customers return to their normal rate of demand, thus causing overstock at the level of their supplier, and thus causing the bullwhip effect and consequently the CFB.

Several studies confirm the positive role that certain digital technologies can play in controlling this operational cause of CFB. Thus, the results of the use of algorithms for forecasting demand in a context of variations in selling prices, allow to conclude on the positive impact that artificial intelligence can have in controlling price variations [24].

In addition, the blockchain also makes it possible to store contractual data that the parties can use to detect price fluctuations, in order to correlate them with fluctuations in demand, which contributes to a better analysis of customer demand [28].

4) Rationing games: The rationing games led by members of a supply chain, and which are at the origin of CFB, can be avoided by the use of several digital technologies. Indeed, in the event of a risk of shortage in the market, some companies increase their orders from their suppliers, thus giving a false image on the level of real demand on the market, and pushing suppliers to increase their level of production and stock. To remedy this situation, the deployment of blockchain technology will give suppliers more visibility relative to the actual level of demand, which will allow them to synchronize their production with respect to final demand, rather than blindly following the rationing games of its customers.

5) Lead times: Several research studies highlight the link between the reduction of lead times and the use of digital 
technologies. Thus, CPS can increase the visibility of physical flows in supply chains and thus improve the availability of information and its sharing. This makes instantaneous data collection and processing possible, reducing production lead times [23], [30]. Additionally, CPS helps in reducing production times by putting feedback loops on production quality [29].

In addition, the speed of data enabled by big data and artificial intelligence also contributes to the reduction of lead times [31].

In addition, the reduction in lead times can be met through the use of cloud computing. Indeed, the sharing of information in real time between the actors of the supply chain, as well as the reduction of data inconsistencies can be ensured by cloud computing [31], [32]. In this sense, the flexibility and scalability of cloud systems provide the necessary infrastructure for simplified information sharing within supply chains, thereby reducing lead times [25].

\section{CONCLUSION}

The results of this research allow to conclude that the use of digital technologies can really impact the operational causes of CFB, and lead to its control. Having said that, and looking at the existing research; it seems that only blockchain, artificial intelligence, cloud computing, cyber-physical systems, IOT, and RFID have a role in this control. On the other hand, we found it difficult to link certain technologies such as additive manufacturing, cyber security, augmented reality, and social media with the reduction of CFB. It would be interesting to empirically prove the positive contribution of the technologies previously mentioned on $\mathrm{CFB}$, and more generally on the overall performance of logistics chains. The diagram below gives a synthetic view of the impact of technologies on CFB (Fig. 8):

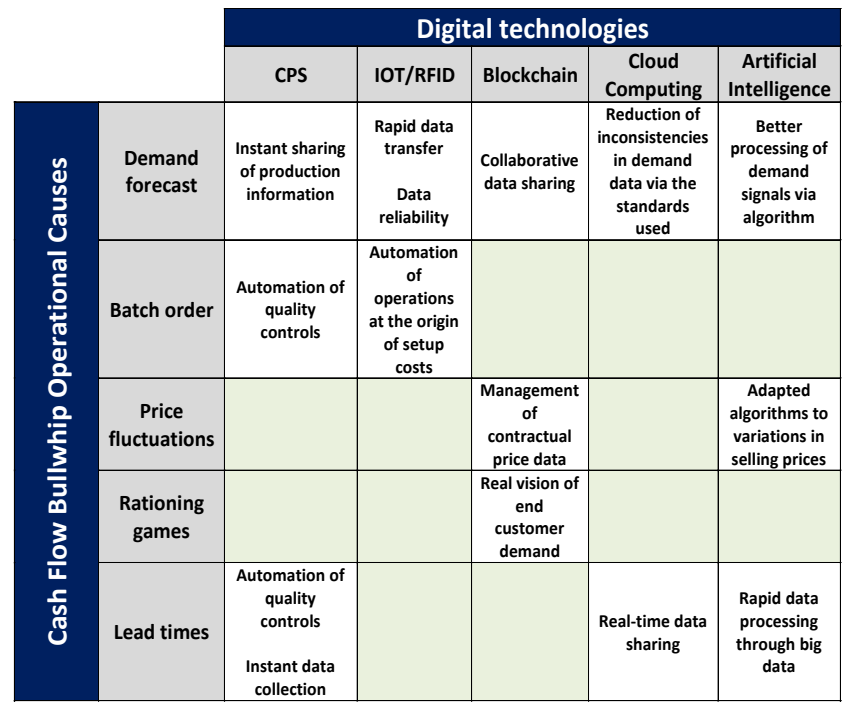

Fig. 8. How Digital Technologies Contribute in the Control of CFB.

\section{REFERENCES}

[1] H. Drissi and M. El ghazali, Audit intene et management des risques, Auditeur. 2018.

[2] M. Fri, F. Fedouaki, K. Douaioui, C. Mabrouki, and E. Semma, "2019, Supply Chain Performance Evaluation Models, State-of-the-Art and
Future Directions,” Oct. 2019, doi: 10.35940/ijeat.A2049.109119.

[3] A. Gacim, H. Drissi, and A. Namir, "Evaluation of the performance of the university information systems: Case of Moroccan universities," Int. J. Adv. Comput. Sci. Appl., vol. 10, no. 6, pp. 224-230, 2019, doi: 10.14569/ijacsa.2019.0100631.

[4] R. Tangsucheeva and V. Prabhu, "Modeling and analysis of cash-flow bullwhip in supply chain," Int. J. Prod. Econ., vol. 145, no. 1, pp. 431447, 2013, doi: 10.1016/j.ijpe.2013.04.054.

[5] H. L. Lee, V. Padmanabhan, and S. Whang, "The Bullwhip Effect in Supply Chains," Sloan Manage. Rev., vol. Vol. 38, no. 3, 1997.

[6] H. Lamzaouek, H. Drissi, and N. El Haoud, "Cash Flow BullwhipLiterature Review and Research Perspectives," Logistics, vol. 5, no. 1, p. 8, 2021, doi: 10.3390/logistics5010008.

[7] D. Y. Liu, S. W. Chen, and T. C. Chou, "Resource fit in digital transformation: Lessons learned from the CBC Bank global e-banking project," Manag. Decis., vol. 49, no. 10, pp. 1728-1742, 2011, doi: $10.1108 / 00251741111183852$.

[8] K. Douaioui, M. Fri, C. Mabroukki, and E. A. Semma, "The interaction between industry 4.0 and smart logistics: Concepts and perspectives," 2018 Int. Colloq. Logist. Supply Chain Manag. LOGISTIQUA 2018, vol. 0021266798, no. April, pp. 128-132, 2018, doi: 10.1109/LOGISTIQUA.2018.8428300.

[9] A. B. and O. A. S. and P. A. P. and N. Venkatraman, "Digital business strategy: toward a next generation of insights," Manag. Inf. Syst. Q., vol. 37, pp. 471-482, 2013, doi: 10.1615/TelecomRadEng.v76.i10.20.

[10] H. Lucas, R. Agarwal, E. Clemons, O. Sawy, and B. Weber, "Impactful Research on Transformational Information Technology: An Opportunity to Inform New Audiences," MIS Q., vol. 37, pp. 371-382, Jun. 2013, doi: 10.25300/MISQ/2013/37.2.03.

[11] F. Schlüter, "Supply Chain Process Oriented Technology-Framework for Industry 4 . 0," no. October, 2017.

[12] R. van Kranenburg and S. Dodson, The Internet of Things: A Critique of Ambient Technology and the All-seeing Network of RFID. Institute of Network Cultures, 2008.

[13] B. Dorsemaine, J. P. Gaulier, J. P. Wary, N. Kheir, and P. Urien, "Internet of Things: A Definition and Taxonomy," Proc. - NGMAST 2015 9th Int. Conf. Next Gener. Mob. Appl. Serv. Technol., no. September, pp. 72-77, 2016, doi: 10.1109/NGMAST.2015.71.

[14] M. Javaid and A. Haleem, "Virtual reality applications toward medical field," Clin. Epidemiol. Glob. Heal., vol. 8, no. 2, pp. 600-605, 2020, doi: 10.1016/j.cegh.2019.12.010.

[15] K. Schwertner, "Digital transformation of business," Trakia J. Sci., vol. 15, no. Suppl.1, pp. 388-393, 2017, doi: 10.15547/tjs.2017.s.01.065.

[16] S. Nakamoto, "Bitcoin: A Peer-to-Peer Electronic Cash System," Cryptogr. Mail. List https//metzdowd.com, Mar. 2009.

[17] P. Tasca and C. J. Tessone, "A Taxonomy of Blockchain Technologies: Principles of Identification and Classification," Ledger, vol. 4, pp. 1-39, 2019, doi: 10.5195/ledger.2019.140.

[18] W. Schroeder, "Germany's Industry 4.0 strategy: Rhine capitalism in the age of digitalisation," Friedrich Ebert Stift., pp. 0-16, 2016, [Online]. Available: https://www.uni-kassel.de/fb05/fileadmin/datas/fb05/FGPoli tikwissenschaften/PSBRD/FES-London_Schroeder_Germanys_ Industrie_4.0_Strategy.pdf.

[19] "Fabrication additive - connaissez-vous la norme française spécifique à cette technologie industrielle innovante? - AFNOR Normalisation." https://normalisation.afnor.org/actualites/fabrication-additiveconnaissez-vous-la-norme-francaise-specifique-a-cette-technologieindustrielle-innovante/ (accessed Nov. 07, 2020).

[20] R. L. V. C. W. Olofson and M. Eastwood, "Big Data: What It Is and Why You Should Care," International Data Corporation (IDC).

[21] S. P.S, N. S, and S. M, "Overview of Cyber Security," Ijarcce, vol. 7, no. 11, pp. 125-128, 2018, doi: 10.17148/ijarcce.2018.71127.

[22] Jay W. Forrester, Industrial Dynamics. MIT Press, Cambridge, Mass, 1961.

[23] S. J. Wang, C. T. Huang, W. L. Wang, and Y. H. Chen, "Incorporating ARIMA forecasting and service-level based replenishment in RFIDenabled supply chain,” Int. J. Prod. Res., vol. 48, no. 9, pp. 2655-2677, 2010, doi: 10.1080/00207540903564983. 
[24] T. O'Donnell, L. Maguire, R. McIvor, and P. Humphreys, "Minimizing the bullwhip effect in a supply chain using genetic algorithms," Int. J. Prod. Res., vol. 44, no. 8, pp. 1523-1543, 2006, doi: 10.1080/00207540500431347.

[25] Y. Yu, R. Q. Cao, and D. Schniederjans, "Cloud computing and its impact on service level: a multi-agent simulation model," Int. J. Prod. Res., vol. 55, no. 15, pp. 4341-4353, 2017, doi: 10.1080/00207543.2016.1251624.

[26] A. Melski, J. Mueller, A. Zeier, and M. Schumann, "Assessing the effects of enhanced supply chain visibility through RFID," 14th Am. Conf. Inf. Syst. AMCIS 2008, vol. 1, no. January 2014, pp. 470-481, 2008.

[27] E. Fleisch and C. Tellkamp, "Inventory inaccuracy and supply chain performance: A simulation study of a retail supply chain," Int. J. Prod. Econ., vol. 95 , no. 3, pp. 373-385, 2005, doi: 10.1016/j.ijpe.2004.02.003.

[28] V. Engelenburg, "Delft University of Technology A Blockchain Architecture for Reducing the Bullwhip Effect A blockchain architecture for reducing the bullwhip effect," 2018, doi: 10.1007/978-3-319-94214-
8.

[29] L. Baur and E. M. Frazzon, "Evaluating the contribution of in-line metrology to mitigate bullwhip effect in internal supply chains," IFACPapersOnLine, vol. 51, no. 11, pp. 1714-1719, 2018, doi: 10.1016/j.ifacol.2018.08.209.

[30] Y. Zhang, L. Zhao, and C. Qian, "Modeling of an IoT-enabled supply chain for perishable food with two-echelon supply hubs," Ind. Manag. Data Syst., vol. 117, no. 9, pp. 1890-1905, 2017, doi: 10.1108/IMDS10-2016-0456.

[31] T. Eggenberger, K. Oettmeier, and E. Hofmann, "Industrializing Additive Manufacturing - Proceedings of Additive Manufacturing in Products and Applications - AMPA2017," Ind. Addit. Manuf. - Proc. Addit. Manuf. Prod. Appl. - AMPA2017, 2018, doi: 10.1007/978-3-31966866-6.

[32] C. Gonul Kochan, D. R. Nowicki, B. Sauser, and W. S. Randall, "Impact of cloud-based information sharing on hospital supply chain performance: A system dynamics framework," Int. J. Prod. Econ., vol. 195, pp. 168-185, 2018, doi: 10.1016/j.ijpe.2017.10.008. 